\title{
Procesamiento de la Cascarilla de Cebada Cervecera por Vía Enzimática para la Obtención de Azúcares Fermentables
}

\author{
Yuliana Cruz-Cardona ${ }^{(1)}{ }^{*}$, Edith Cadena-Chamorro ${ }^{(2)}$, y Julio C. Arango-Tobón (2) \\ (1) Facultad de Ciencias, Universidad Nacional de Colombia, Carrera 65 № 59â-110, Bloque 19A, Medellín, \\ Colombia. (e-mail: ymcruzc@unal.edu.co)

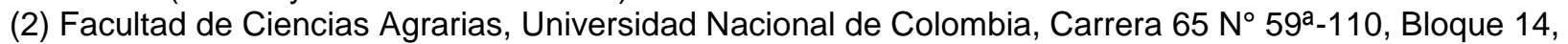 \\ Medellín, Colombia. (e-mail: emcadenac@unal.edu.co; jcarango@unal.edu.co)
}

* Autor a quien debe ser dirigida la correspondencia

Recibido Sep. 27, 2018; Aceptado Dic. 10, 2018; Versión final Feb. 15, 2019, Publicado Ago. 2019

\begin{abstract}
Resumen
Se presenta un estudio orientado a promover la sacarificación de la cascarilla de cebada a través de la aplicación de complejos enzimáticos comerciales. La cascarilla de cebada cervecera corresponde a un residuo lignocelulósico potencial para su introducción en procesos de biorrefinería dada su composición química y alto volumen de material residual. En este contexto, Viscozyme L, Celluclast 1.5L, Hemicellulase y Xylanase fueron evaluados en función de la dosificación con el objetivo de maximizar la respuesta en azúcares reductores. La hidrólisis enzimática presentó un efecto significativo obteniéndose los mejores resultados para el complejo Viscozyme L con una máxima liberación de glucosa de $16.9 \mathrm{~g} / \mathrm{L}$. Los resultados dan viabilidad a la implementación del uso del complejo Viscozyme L., permitiendo procesos más limpios y energéticamente eficientes.
\end{abstract}

\section{Processing of Brewer's Spent Grain by Enzymatic Means to Obtain Fermentable Sugars}

\begin{abstract}
A study aimed at promoting the saccharification of brewer's spent grain through the application of commercial enzymatic complexes is presented. Brewer's spent grain corresponds to a potential lignocellulosic residue for its introduction in biorefinery processes given its chemical composition and high volume of residual material. In this context, Viscozyme L, Celluclast 1.5L, Hemicellulase and Xylanase were evaluated according to the dosage in order to maximize the response in reducing sugars. Enzymatic hydrolysis presented a significant effect, obtaining the best results for the Viscozyme L complex with a maximum glucose release of $16.9 \mathrm{~g} / \mathrm{L}$. The results indicate that the implementation of the Viscozyme $L$ complex is possible, allowing cleaner and energy efficient processes.
\end{abstract}

Keywords: lignocellulosic waste; hydrolytic enzymes; reducing sugars 


\section{INTRODUCCIÓN}

La producción de cerveza corresponde principalmente al procesamiento de cebada malteada e incluye etapas de maceración, cocción, fermentación y maduración. La maceración, tiene como objetivo la activación enzimática, extracción de sustancias fenólicas, azúcares simples y compuestos nitrogenados requeridos para el siguiente proceso fermentativo. Posterior a la maceración se realiza una filtración para separar todo el material orgánico del mosto, obteniéndose como residuo la cascarilla de cebada malteada, conocida como Brewer's spent grain (BSG) (Figura 1), representando el $85 \%$ de los subproductos generados en esta industria (Mussatto et al., 2013). A nivel mundial, se ha reportado un promedio de 34 millones de toneladas de BSG (Xiros y Christakopoulos, 2012). En Colombia, la industria cervecera se caracteriza por un nivel elevado de concentración industrial, diferenciación de productos y explotación de economías de escala (DNP, s.f.). Para 2017, la producción de cerveza en litros fue de 2189 millones (Barth, 2018) liderando el mercado de licores y bebidas del país con un consumo anual de un millón de hectolitros (ProChile, 2011). Así, esta biomasa residual, por su disponibilidad y composición se convierte en materia prima potencial para otros procesos como biocombustibles líquidos y gaseosos (Aliyu y Bala, 2013; Zhang y Zang, 2016; Wilkinson et al., 2015); como sustrato para la obtención de compuestos bioactivos, antioxidantes, monosacáridos, oligosacáridos, xilitol, arabitol, ácidos orgánicos, proteínas, y lípidos, entre otros productos de alto valor agregado (McCarthy et al., 2013; Liang y Wan, 2015; Lynch et al., 2016). Actualmente, los destinos más comunes de la cascarilla de cebada malteada corresponden a suplementos en dieta animal presentando muchos beneficios nutricionales, y compostaje para la obtención de abonos para el mejoramiento de suelos (Mussatto et al., 2014).

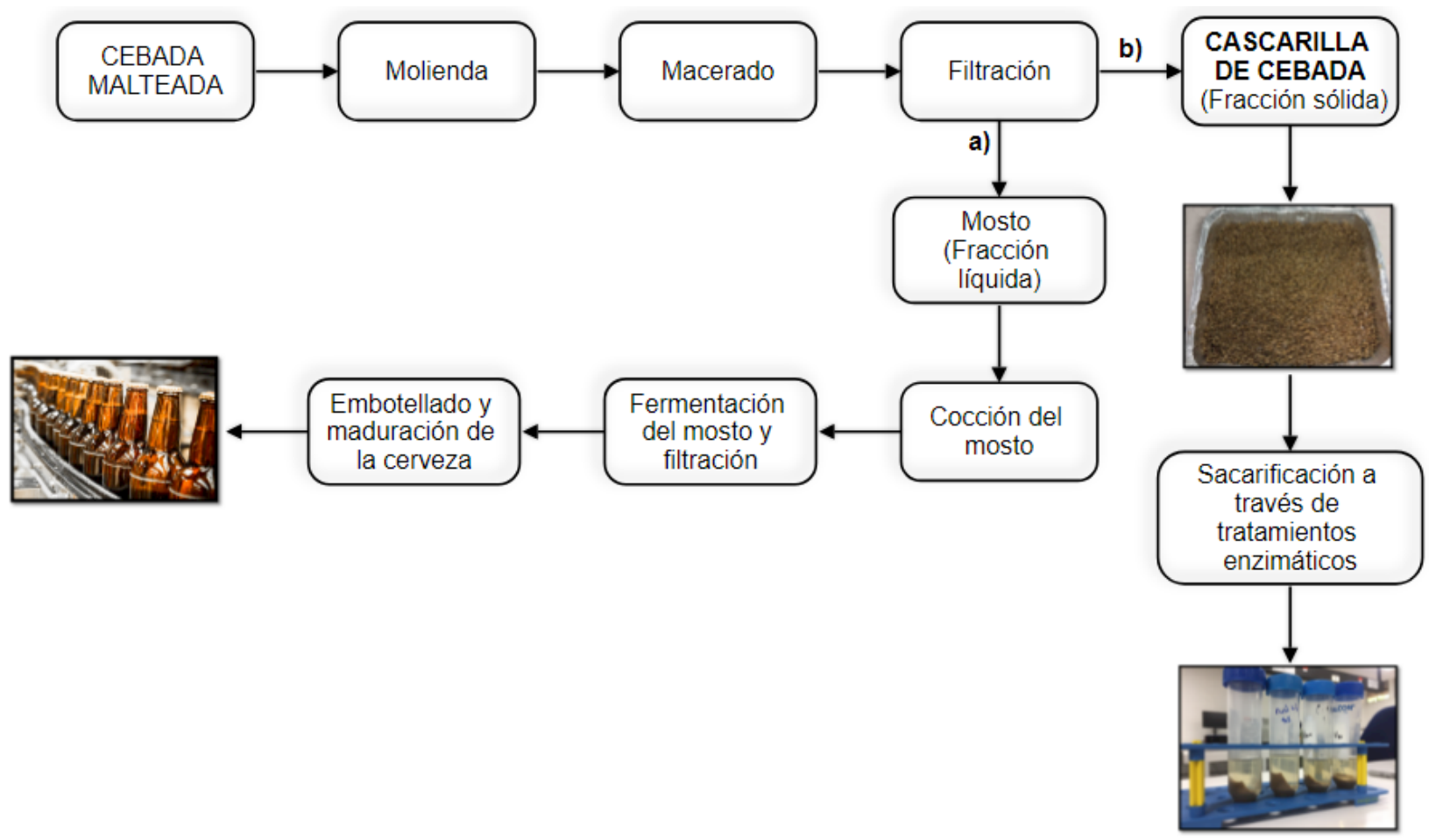

Fig. 1: Proceso cervecero y aprovechamiento de Cascarilla de Cebada Cervecera.

El potencial económico del brewer's spent grain radica en sus componentes lignocelulósicos principalmente celulosa, hemicelulosa y lignina, y otros compuestos minoritarios como extractivos hidrofílicos y lipofílicos que cumplen una función no estructural. En general, el fraccionamiento de las cuatro categorías en todo material lignocelulósico permite obtener una amplia gama de productos químicos como biocombustibles, productos bioquímicos industriales y biomateriales de importancia industrial bajo el concepto de biorefinería (ruta promisoria para la creación de una economía de base biológica) que es análogo a la refinería de petróleo la cual toma hidrocarburos para la obtención de derivados comerciales (Ghatak, 2011; Venkata Mohan et al., 2016). Específicamente, en el BSG el contenido de celulosa oscila entre el 15 y $26 \%$, hemicelulosa $22-40 \%$, lignina 4 - 28\%, proteína 14 - 24\%, almidón 2 - 8\%, cenizas 2 - 5\%, extractivos 6 - 11\% (Mussatto et al., 2013; McCarthy et al., 2013). La variabilidad en composición está ligada a diversos factores entre ellos los correspondientes al eslabón primario o prácticas agronómicas del cultivo de cebada, variedad, condiciones bioclimáticas, edáficas y de ubicación en altitud, en procesos de transformación el tipo de malteado, secado y molienda, en el proceso cervecero a nivel artesanal o industrial influye el tipo de maceración sea sencilla o escalonada (Mussatto et al., 2006). 
En el caso de biocombustibles, la bioconversión de la matriz polimérica o lignocelulósica del BSG requiere de pretratamientos térmicos, físicos y/o químicos para producir azúcares fermentables, seguido de una fermentación. El uso de álcalis, ácidos y solventes orgánicos son los pretratamientos más frecuentes por la eficiencia en la liberación de azúcares, sin embargo, la aplicación de altas concentraciones imposibilita la recuperación de sólidos, generan compuestos inhibitorios para los procesos fermentativos e incrementan costos de producción (Ravindran et al., 2018). La ruta termoquímica, específicamente la pirólisis permite obtener y controlar de manera más sistemática numerosos productos químicos (Borel et al., 2018), aunque hay restricciones ya que son tecnologías con altos requerimientos energéticos. Por otro lado, las conversiones por ruta biológica son preferidas a la química por ser más selectivas y promisorias para la consecución de etapas más limpias, aunque debido la complejidad de la biomasa lignocelulósica exige múltiples procesos para lograr eficiencia en las operaciones; adicionalmente, la lentitud de las reacciones biotecnológicas es una limitación y se requieren optimizar los tiempos de hidrólisis, rendimientos y productividad (Alvira et al., 2010). En este contexto, el uso de aplicaciones enzimáticas para la degradación de materia orgánica ha sido ampliamente investigado en la producción de biocombustibles; debido a la naturaleza de los residuos orgánicos de fuente agro-industrial se han reportado evaluaciones de diferentes enzimas hidrolíticas con actividad $\beta$-glucanasa, celulasa, hemicelulasa, xilanasa, proteasas, lipasas (Wilkinson et al., 2017; Ravindran y Jaiswal, 2016) y enzimas con actividad ligninolítica como peroxidasas y lacasas (El-Naggar et al., 2014). La accesibilidad a la matriz polimérica se convierte en un desafío, entre las propiedades estructurales que dificultan el proceso de hidrólisis enzimática se menciona el grado de polimerización, hidrofobicidad, cristalinidad, área superficial, entre otras.

De acuerdo a Niemi et al. (2012), la aplicación de complejos enzimáticos de actividad hidrolítica genera una solubilidad de carbohidratos hasta del $48 \%$ en combinación con pretratamientos físicos en la cascarilla de cebada. Así, la presente investigación se enfoca al aprovechamiento de subproductos lignocelulósicos de la industria cervecera para maximizar el rendimiento de azúcares fermentables a través de combinaciones enzimáticas en función de concentración y tiempo de proceso con proyección a minimización de costos operacionales para una posterior obtención de bioetanol o aplicación en biocombustibles. Esto permitirá el fortalecimiento de dicha industria al aplicar procesos integrales que promuevan la generación de productos de valor agregado a través del aprovechamiento de residuos de un proceso, y en consecuencia contribuir al desarrollo sostenible.

\section{MATERIALES Y MÉTODOS}

En esta sección se describe la preparación del residuo lignocelulósico proveniente de la industria cervecera, las condiciones de operación en los tratamientos enzimáticos y sus correspondientes metodologías de caracterización.

\section{Preparación y caracterización del material lignocelulósico}

La cascarilla de cebada (Brewer's spent grain) fue suministrada por Inducerv S.A.S. Posterior a un secado por convección forzada a $70^{\circ} \mathrm{C}$ durante 12 horas, la muestra fue triturada en su totalidad en un molino de martillos con un tamiz de $0.5 \mathrm{~mm}$ de apertura de malla, finalmente se esterilizó con vapor de agua a $130{ }^{\circ} \mathrm{C}$ durante $3 \mathrm{~min}$. La caracterización química se realizó en términos de cenizas, minerales (Espectrometría de Absorción Atómica y Espectrofotometría U.V-VIS), contenido de celulosa y hemicelulosa (Tappi T 203, 2009), contenido de lignina insoluble por hidrólisis acida (Tappi T 222, 1999) y proteína cruda (Kjeldahl).

\section{Tratamientos enzimáticos en la cascarilla de cebada}

Las enzimas evaluadas para el proceso hidrolítico corresponden a Viscozyme $L$ (complejo multienzimático que contiene $\beta$-glucanasa, celulasa, hemicelulasa y xilanasa; derivado de $A$. aculeatus, actividad $100 \mathrm{FBG} / \mathrm{g}$ ); Celluclast 1.5L (derivado de $T$. reesei, actividad $\geq 700 \mathrm{U} / \mathrm{g}$ ); Hemicelulasa (derivado de $A$. niger 0.3-3.0 U/mg); y Xilanasa (derivado de $A$. oryzae $\geq 2500 \mathrm{U} / \mathrm{g}$ ). Los tratamientos enzimáticos se efectuaron en un shaker bajo agitación constante a $250 \mathrm{rpm}$ (MaxQ 4450, Alemania), pH 5.0 (buffer acetato $0.1 \mathrm{M}$ ), $50{ }^{\circ} \mathrm{C}$ y a una concentración de sustrato del $5 \% \mathrm{p} / \mathrm{v}$.

Las evaluaciones enzimáticas se realizaron en función del tipo de enzima y dosificación (unidades de enzima por gramo de cascarilla en base seca) acorde a las especificaciones de la Tabla 1 . El tratamiento $\mathrm{C}-\mathrm{H}: \mathrm{X}$ se realizó en dos etapas; posterior a la aplicación de Celluclast 1.5L se adicionó una mezcla de Hemicelulasa y Xilanasa; y el tratamiento H-V incluye la aplicación secuencial de Hemicelulasa y Viscozyme L. Finalizado el tiempo de hidrólisis las muestras fueron centrifugadas a $10000 \mathrm{rpm}$ a $4{ }^{\circ} \mathrm{C}$ durante 10 minutos con el objetivo de extraer el sobrenadante para posterior análisis de azúcares reductores. 
Tabla 1: Tratamientos enzimáticos aplicados sobre la cascarilla de cebada.

\begin{tabular}{cll}
\hline Tratamiento & \multicolumn{1}{c}{ Enzimas } & \multicolumn{1}{c}{ Dosificación y tiempo de hidrólisis } \\
\hline Vosis $(\mathrm{U} / \mathrm{g}): 2,10,18,26,34,42,50$. \\
Viscozyme L & $\begin{array}{l}\text { Tiempo: } 5 \mathrm{~h} \\
\text { Celluclast 1.5L }\end{array}$ & $\begin{array}{l}\text { Dosis }(\mathrm{U} / \mathrm{g}): 2,10,18,26,34,42,50 . \\
\text { Tiempo: } 5 \mathrm{~h}\end{array}$ \\
\hline $\mathrm{C}-\mathrm{H}: \mathrm{X}$ & Celluclast 1.5L & $\begin{array}{l}\text { Dosis }(\mathrm{U} / \mathrm{g}): 42 \\
\text { Tiempo: } 5 \mathrm{~h}\end{array}$ \\
\cline { 2 - 3 } & $\begin{array}{ll}\text { Xylanase y } \\
\text { Hemicellulase }\end{array}$ & $\begin{array}{l}\text { Dosis }(\mathrm{U} / \mathrm{g}): 10: 530: 1550: 25 \\
\text { respectivamente. } \\
\text { Tiempo: } 2 \mathrm{~h}\end{array}$ \\
$\mathrm{H}$ & Hemicellulase & $\begin{array}{l}\text { Dosis }(\mathrm{U} / \mathrm{g}): 30 \\
\text { Tiempo: } 2 \mathrm{~h}\end{array}$ \\
\cline { 2 - 3 } $\mathrm{H}-\mathrm{V}$ & Viscozyme L & $\begin{array}{l}\text { Dosis }(\mathrm{U} / \mathrm{g}): 50 \\
\text { Tiempo: } 5 \mathrm{~h}\end{array}$ \\
& &
\end{tabular}

\section{Estudio cinético y evaluación del tiempo de hidrólisis}

Para la aplicación del complejo enzimático Viscozyme $L(V)$, se evaluó el efecto de concentración del sustrato (en un rango de 0.1 a 2.5 gramos de cascarilla de cebada, $50 \mathrm{U} / \mathrm{g}$ de Viscozyme L, 5 horas) y del tiempo (0.5 a 10 horas, $25 \mathrm{U} / \mathrm{g}$ de Viscozyme L, sustrato al $5 \% \mathrm{p} / \mathrm{v}$ ) sobre la velocidad de hidrólisis a través de parámetros cinéticos Km y Vmax, según el modelo de Michaelis-Menten y posterior linealización mediante el método de Lineweaver-Burk (Ravindran et al., 2019). La hidrólisis se realizó a pH 5.0 y $50^{\circ} \mathrm{C}$.

\section{Determinación de azúcares reductores}

El efecto de las aplicaciones enzimáticas fue analizado en función de la liberación de azucares reductores utilizando el método del ácido 3,5-dinitrosalicílico (DNS). Se utilizó una curva de calibración en función del estándar glucosa en concentraciones de 0 a $2 \mathrm{~g} / \mathrm{L}\left(X=1.58 \mathrm{y}+0.285, \mathrm{R}^{2}=0.9983\right.$, donde $\mathrm{Y}$ es la absorbancia y X los g/L de glucosa. La absorbancia fue determinada en un espectrofotómetro UV-Vis (UV-2550, Shimadzu, Japón) a una longitud de onda de $540 \mathrm{~nm}$ (Miller, 1959). La determinación se realizó por triplicado. Las diferencias significativas entre cada tratamiento se analizaron mediante análisis de varianza (ANOVA) y prueba de Tukey para comparación de medias con un nivel de significancia del $5 \%$ utilizando el software Statgraphics versión XVI.I. El porcentaje de hidrólisis correspondiente a la equivalencia entre unidades de azúcares reductores con base a sustrato en peso seco se realizó con la ecuación 1:

$$
\% \text { Hidrólisis }=\frac{\text { Azúcares reductores }\left(\frac{g}{\mathrm{~L}}\right)}{\text { Concentración de sustrato }\left(\frac{\mathrm{g}}{\mathrm{L}}\right)} * 100
$$

\section{RESULTADOS Y DISCUSIÓN}

Se presentan los resultados de los diferentes tratamientos enzimáticos sobre la cascarilla de cebada que corresponde al residuo lignocelulósico de la industria cervecera; y el respectivo efecto de las combinaciones de las enzimas Viscozyme L, Celluclast 1.5L, Hemicelulasa, y Xilanasa con dosificación y tiempo sobre la liberación de azúcares reductores expresada en g/L de glucosa.

La cascarilla de cebada presentó un contenido de celulosa equivalente al $26.56 \pm 2.03 \%$, este porcentaje se encuentra entre los rangos reportados por otros autores (Mussatto., 2014). El alto contenido de celulosa lo hace un material viable para el tratamiento hidrolítico con enzimas de actividad celulasa. Con relación a los contenidos de hemicelulosa, lignina y cenizas, se presentaron valores de $31.92 \pm 2.03,22.31 \pm 0.00$ y $2.60 \pm$ 0.04 respectivamente. En su composición también se encuentran extraíbles del tipo hidrosolubles y lipofílicos que pueden oscilar en un 16.5\%. En la caracterización se destaca el alto contenido de lignina, al ser un heteropolímero tridimensional amorfo, principalmente construido con monómeros de base estructural correspondientes a unidades de fenil-propano, se convierte en el principal obstáculo en los procesos de degradación de la holocelulosa, es catalogado como agente segmentante con funciones estructurales y antimicrobianas. Al respecto, se han reportado aplicaciones exitosas con enzimas ligninolíticas, en concreto el sistema lacasa mediador ha permitido una eficaz degradación de lignina en materiales lignocelulósicos (Raj y Krishnan, 2018), sin embargo en Brewer's spent grain los estudios son escasos. En cuanto al análisis de minerales y proteína, presentó un contenido porcentual de calcio, fósforo y magnesio de $0.11,0.30,0.14$ respectivamente; la concentración de potasio fue de $636 \mathrm{ppm}$ y el contenido de proteína cruda de $18.7 \%$. La cantidad presente de proteína y minerales en BSG es significativa (Xiros et al., 2008), lo que convierte a este subproducto es una importante fuente de nitrógeno y elementos traza. Sumado a su alto contenido en 
polisacáridos, capacidad de retención de agua y porosidad, el BSG se propone como un medio de cultivo complejo apto para el crecimiento de microrganismos y producción de metabolitos de interés o de enzimas hidrolíticas (Xiros y Christakopoulos, 2012).

La Figura 2, presenta la efectividad hidrolítica de los tratamientos enzimáticos de Vizcozyme L (V) y Celluclast $1.5 \mathrm{~L}(\mathrm{C})$ a diferentes dosis de enzimas. Se observa que a mayor dosificación se obtiene un efecto superior en la liberación de azúcares con respecto al tratamiento control. La aplicación de Viscozyme L genera una mayor respuesta en la sacarificación, en contraste a Celluclast $1.5 \mathrm{~L}$, el resultado confirma el requerimiento de un complejo enzimático apto para una matriz lignocelulósica. El análisis de varianza (ANOVA), indica que los factores dosis y tipo de enzima son estadísticamente significativos a un nivel de confianza del $95 \%(p \leq 0.05)$. El máximo valor de glucosa obtenido fue de $16.5 \mathrm{~g} / \mathrm{L}$ (313.5 mg/g BSG) para Viscozyme L, siendo un resultado superior al de otras investigaciones que presentaron tratamientos térmicos previos a la hidrólisis enzimática y que no incluyen tratamientos químicos o termo-mecánicos; Macheiner et al. (2003), reporta una concentración de glucosa de $150 \mathrm{mg} / \mathrm{g}$ BSG aproximadamente al aplicar Ceremix plus MG sobre BSG posterior a un pretratamiento microondas a $160^{\circ} \mathrm{C}$ por 10 minutos. Ravindran et al., 2018, reporta azúcares reductores de $194 \mathrm{mg} / \mathrm{g} \mathrm{BSG}$, con un pretratamiento de steam explosión y posterior hidrólisis con celulasa y hemicelulasa.

Es importante mencionar que para la degradación de celulosa en monómeros de glucosa como unidades básicas se requiere de una acción sinérgica entre las enzimas exo-1,4- $\beta$-glucanasa, endo-1,4- $\beta$-glucanasa y la $\beta$-glucosidasa (De Vries y Visser, 2001; Van Dyk y Pletschke, 2012). Las endoglucanasas realizan cortes al azar en el interior de la celulosa amorfa, generando oligosacáridos de varias longitudes y, en consecuencia, nuevos extremos de cadena. Las exoglucanasas actúan de una manera progresiva en los extremos reductores y no reductores de las cadenas del polisacárido, liberando glucosa o celobiosa. Las $\beta$-glucosidasas hidrolizan las celodextrinas solubles y la celobiosa a glucosa (Jeoh et al., 2017). En consecuencia al utilizar un complejo de actividad celulasa se esperaba un resultado favorable, pero la baja efectividad de Celluclast 1.5 $\mathrm{L}$ puede deberse a una posible limitación de accesibilidad al sustrato por el alto contenido de lignina, la presencia de inhibidores, por las características estructurales intrínsecas correspondientes al área superficial del residuo a procesar, grado de porosidad, cristalinidad o por características hidrofóbicas. La dosificación de Celluclast $1.5 \mathrm{~L}$ sobre la cascarilla de cebada no genera un efecto significativo sobre la liberación de azucares reductores durante 5 horas de tratamiento a pH 5.0 y a $50 \stackrel{\circ}{\circ}$.

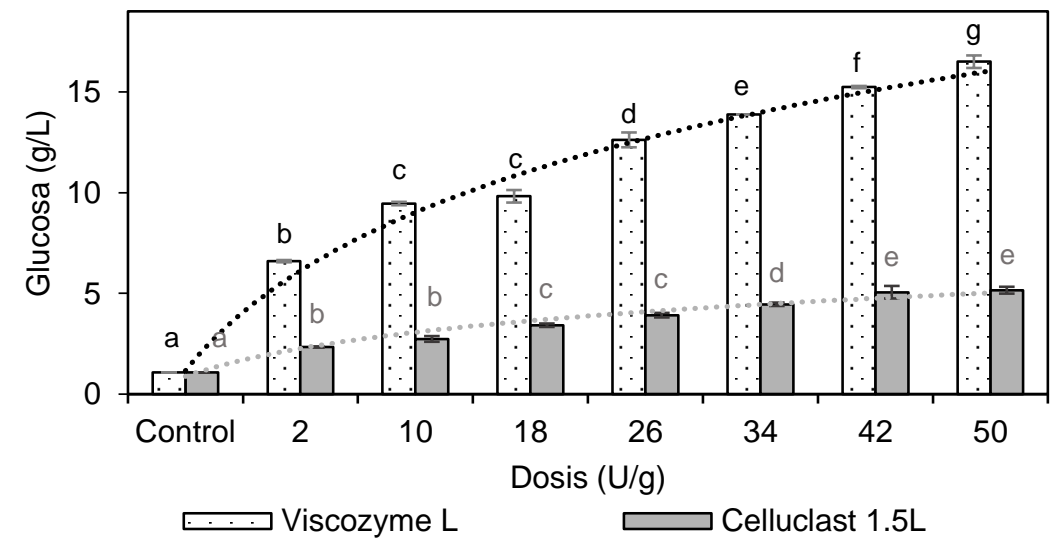

Fig. 2: Efecto enzimático de Viscozyme $L$ y Celluclast 1.5L sobre el proceso de sacarificación en la cascarilla de cebada cervecera. *Letras diferentes indican diferencia estadísticamente significativa entre las dosis de cada enzima $(p \leq 0,05)$.

El tratamiento que corresponde a la aplicación secuencial de Celluclast 1.5L, Hemicelulasa y Xilanasa (C$\mathrm{H}: \mathrm{X}$ ), se realizó con una dosis de Celluclast $1.5 \mathrm{~L}$ de $42 \mathrm{U} / \mathrm{g}$, seleccionada a partir de los resultados presentados en la Figura 2. La expectativa con las hemicelulasas y xilanasas se enfoca al alto contenido de hemicelulosa de la cascarilla de cebada. La hemicelulosa es un polímero no cristalino, con menor grado de polimerización que la celulosa, inferior a 200 monómeros, que presenta alto grado de ramificación (Cabeza et al., 2018). Forma parte de la pared celular, recubriendo la superficie de las fibras de celulosa, a su vez los xilanos corresponden al componente mayoritario de la hemicelulosa (Zabed et al., 2016). Así, las combinaciones de hemicelulasa y xilanasa se justifican por la presencia del sustrato.

Los resultados de los tratamientos $\mathrm{C}-\mathrm{H}: \mathrm{X}$ en las relaciones 10:5, 30:15, 50:25 U/g correspondientes a las denominaciones $\mathrm{CHX} 1, \mathrm{CHX} 2$ y $\mathrm{CHX} 3$, respectivamente, se presentan en la Figura 3. La máxima concentración de glucosa equivalente a $6.53 \mathrm{~g} / \mathrm{L}$ se obtuvo para la aplicación $\mathrm{CHX3}$. El análisis de varianza señala que la relación $\mathrm{C}-\mathrm{H}: \mathrm{X}$ presenta un efecto significativo en la respuesta con respecto al control $(\mathrm{p} \leq 0.05)$. En $\mathrm{C}-\mathrm{H}: \mathrm{X}$, la aplicación inicial de celulasa además de la hidrólisis generaría un efecto de modificación 
morfológica en el material lignocelulósico, es decir un incremento en el área superficial haciendo posible una mayor actuación secuencial de hemicelulasas y xilanasas en la matriz, junto con actividad celulasa residual. Al no presentarse una respuesta marcada en la generación de azúcares reductores con respecto a Viscozyme $\mathrm{L}$, se intuye que en la primera etapa se puede presentar una inhibición por presencia de celobiosa y glucosa tanto para celulasas como hemicelulasas. El tiempo de tratamiento para celulasa fue de 5 horas en consecuencia una alternativa puede corresponder a una reducción en el tiempo de hidrólisis. Por otro lado, también se evalúa el efecto sinérgico de la enzima hemicelulasa con el complejo Viscozyme L (tratamiento HV). La hemicelulosa se encuentra más disponible para una hidrolisis enzimática directa, posteriormente el complejo Viscozyme $L$ que incluye actividades arabinasa, celulasa, $\beta$-glucanasa, hemicelulasa, y xilanasa, presentaría un efecto superior al obtenido, sin embargo el resultado no fue el esperado, y se puede correlacionar con una posible inhibición por producto causada por la primera acción de la enzima hemicelulasa. La combinación H-V reporta un valor inferior en $2.5 \mathrm{~g} / \mathrm{L}$ unidades de glucosa al obtenido con Viscozyme L, siendo este último el complejo más idóneo para procesos hidrolíticos en materiales lignocelulósicos, y también ha sido reportado por otros investigadores (Duarte et al., 2004).

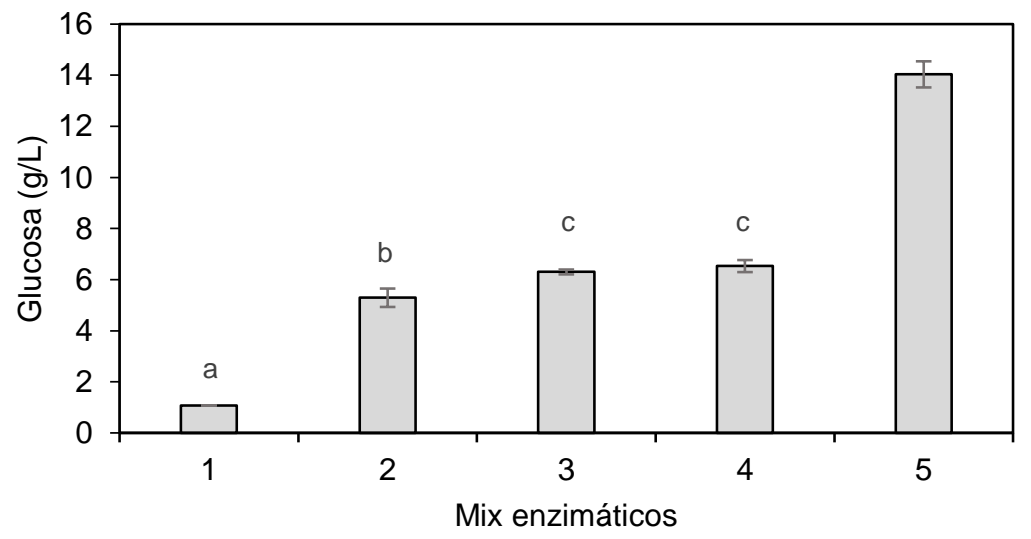

Fig. 3: Efecto enzimático de los tratamientos $\mathrm{C}-\mathrm{H}: \mathrm{X}$ y H-V sobre el proceso de sacarificación en la cascarilla de cebada cervecera. * Letras diferentes indican diferencia estadísticamente significativa entre las dosis de cada enzima $(p<0,05)$.

En aplicaciones enzimáticas, es primordial optimizar procesos con el objeto de minimizar costos en relación a dosificación, materia prima y tiempo de procesos en función del máximo rendimiento o productividad, para este fin se realizan cinéticas enzimáticas. Así, para Viscozyme $L$ se determinó el efecto del sustrato, acorde a los lineamientos de la cinética de Michaelis-Menten se debe garantizar saturación de las enzimas por el sustrato para alcanzar la velocidad máxima de una reacción. En la Figura 4a, se observa que la concentración de glucosa aumenta con respecto a la cantidad de sustrato con una pendiente marcada hasta $2.0 \mathrm{~g}$ para una posterior estabilización o un descenso a $2.5 \mathrm{~g}$. Los parámetros cinéticos determinados por la linealización Lineweaver-Burk presentan valores de $2.98 \mathrm{~g} / \mathrm{L}$ y $0.048 \mathrm{~g} / \mathrm{L}$ min correspondientes a $\mathrm{Km}$ y Vmax, respectivamente. Con respecto al tiempo, el tratamiento enzimático genera mayores resultados a medida que incrementa el tiempo del proceso con respecto al control K1 (BSG sin hidrolizar) y control K2 (solución buffer más dosis de enzima); a las 7 horas se obtiene un punto máximo de $16.90 \mathrm{~g} / \mathrm{L}$ y posteriormente se aprecia un descenso a $14.61 \mathrm{~g} / \mathrm{L}$ de glucosa que puede ser debido a inhibición por producto (Figura 4b).

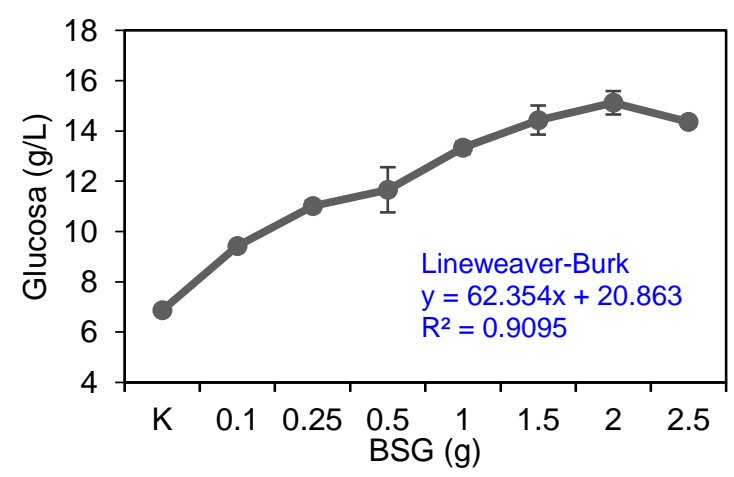

a)

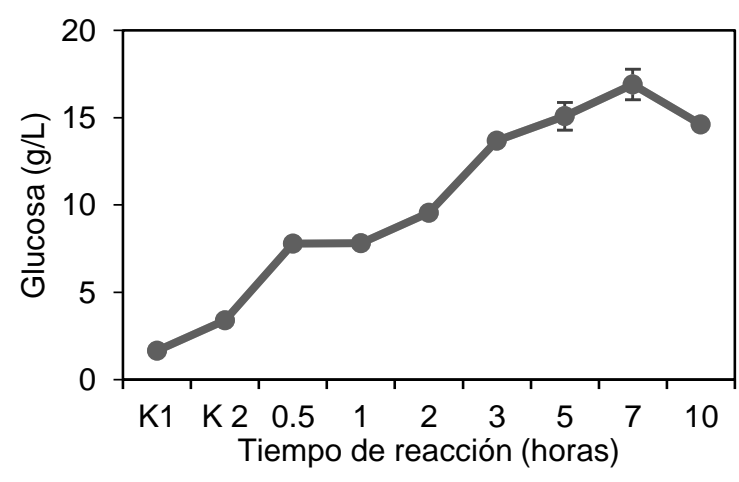

b)

Fig. 4: Hidrólisis enzimática Viscozyme L: a) Efecto del sustrato con 50 U/g dosis de enzima, b) Efecto del tiempo del tratamiento con $25 \mathrm{U} / \mathrm{g}$ dosis de enzima. 
Los pretratamientos fisicoquímicos son comúnmente empleados por el mayor rendimiento de azúcares reductores en comparación a los biológicos o enzimáticos. Sin embargo, de estos últimos se obtienen resultados promisorios al combinarlos con pretratamientos físicos y térmicos, siendo merecedores de un estudio adecuado para optimizar sus condiciones y así aplicarlos en un proceso final, limpio y eficiente del residuo agroindustrial. Diversos pretratamientos y combinaciones enzimáticas han sido reportados en la cascarilla de cebada como casos exitosos; Macheiner et al. (2003), destacan la hidrólisis con $\mathrm{HCl}(0.1 \mathrm{M})$ asistida con radiación microondas cuyo efecto incremento los azúcares reductores obteniéndose valores de $359.2 \mathrm{mg} / \mathrm{g}$ BSG en 10 minutos de hidrólisis a $160^{\circ} \mathrm{C}$. En otros pretratamientos incluyen reducción de tamaño de partícula y extrusión húmeda en dos ciclos a $160^{\circ} \mathrm{C}$. Posteriormente tras la aplicación de CelluPract AL7 reportaron la obtención $160 \mathrm{mg} / \mathrm{g}$ BSG de azucares reductores. En contraste, Pierre et al., (2011), obtuvieron $240 \mathrm{mg} / \mathrm{g} \mathrm{BSG}$ de azúcares reductores con un pretratamiento termomecánico a $163^{\circ} \mathrm{C}$ seguido de hidrólisis con Celluclast 1.5L.

El pretratamiento térmico permite una alteración estructural de los biopolímeros que luego se reorganizan permitiendo un mejor acceso de las enzimas hidrolíticas a los enlaces glucosídicos (Macheiner et al., 2003). La reducción del tamaño de partícula mejora el rendimiento de la hidrólisis de celulosa; la molienda aumenta las regiones amorfas de la celulosa (Zhao et al., 2006). Además, la degradación mecánica expone las partes internas del grano, cuyas paredes celulares son más fáciles de hidrolizar. Esto aumenta la cantidad de carbohidratos solubles en agua y mejora la velocidad de reacción inicial (Niemi et al., 2012). En cuanto a la hidrólisis con ácidos y álcalis diluidos, pueden ser beneficiosos para mejorar el rendimiento de glucosa durante la hidrólisis de celulosa al eliminar parcialmente la fracción de hemicelulosa y lignina, pero implica lavados secuenciales sobre el material hidrolizado para eliminar subproductos y evitar efectos inhibidores en la reacción enzimática o en la fermentación (Mussatto et al, 2008). Según los resultados alcanzados en esta investigación, la estrategia implementada avala la combinación de los pretratamientos físico, térmico y enzimático como una metodología apta para la obtención de azúcares fermentables, sin comprometer los compuestos generados para futuras fases del bioproceso.

\section{CONCLUSIONES}

Con el procedimiento desarrollado se espera maximizar la liberación de azúcares reductores a partir de la cascarilla de cebada cervecera evaluando las condiciones de concentración de sustrato, dosis de enzima y tiempo de hidrólisis. El pretratamiento térmico y la molienda han facilitado la reacción de las diferentes aplicaciones enzimáticas. El máximo valor de glucosa obtenido fue de $16.90 \mathrm{~g} / \mathrm{L}$ (equivalente a $321.11 \mathrm{mg} / \mathrm{g}$ BSG). Las condiciones óptimas de este resultado fueron halladas para el complejo enzimático Viscozyme $L$ con una dosis de $25 \mathrm{U} / \mathrm{g}, \mathrm{pH} 5.0,50^{\circ} \mathrm{C}$, tiempo de hidrólisis 7 horas y una consistencia de BSG del $5 \% \mathrm{p} / \mathrm{v}$.

El aprovechamiento de la cascarilla de cebada cervecera para la obtención de bioetanol es factible a través de sacarificaciones enzimáticas que incluyan complejos enzimáticos. Para un mayor rendimiento de azúcares reductores, se deben evaluar otros tratamientos biológicos más específicos que degraden la matriz lignocelulósica, como es el caso de la lignina y la pectina que obstaculizan la hidrólisis de la celulosa y hemicelulosa. La sinergia e interacción de los complejos enzimáticos y/o sistemas biológicos deben ser evaluados en función de potenciar el rendimiento de azúcares fermentables y la eficiencia del proceso.

\section{AGRADECIMIENTOS}

Los autores agradecen a la Universidad Nacional de Colombia por financiación del proyecto "Evaluación de hidrólisis enzimáticas de residuos orgánicos para la producción de biocombustibles". Código Hermes: 36096, a través de la Convocatoria Nacional de proyectos para el fortalecimiento de la investigación, creación e innovación de la Universidad Nacional de Colombia 2016-2018.

\section{REFERENCIAS}

Aliyu, S. y M. Bala, Brewer's Spent Grain: A Review of its Potentials and Applications, doi: 10.4314/ajb. v10i3, African Journal of Biotechnology, 10(3), 324-331 (2013)

Alvira, P., E. Tomás-Pejó, M. Ballesteros y M. J. Negro, Pretreatment technologies for an efficient bioethanol production process based on enzymatic hydrolysis: A review, Bioresource Technology, 101(13), 4851-4861 (2010)

Barth, S. J., (Barth-Haas Group), The Barth Report 2017/2018, Nuremberg-Alemania (2018)

Borel, L. D., T. S. Lira y otros tres autores, Pyrolysis of Brewer's Spent Grain: Kinetic Study and Products Identification, Industrial Crops and Products, 121, 388-395 (2018)

Cabeza, A., C. Piqueras, F. Sobrón y J. García-Serna, Modeling of Biomass Fractionation in a Lab-Scale Biorefinery: Solubilization of Hemicellulose and Cellulose from Holm Oak Wood using Subcritical Water, doi.org/10.1016/j.biortech.2015.09.063, 200, Bioresource Technology, 90-102 (2018) 
De Vries, R. P. y J. Visser, Aspergillus Enzymes involved in Degradation of Plant Cell Wall Polysaccharides, Microbiology and Molecular Biology Reviews, 65, 497-522 (2001)

Departamento Nacional de Planeación (DNP), Cerveza, malta y licores, en línea, https://bit.ly/2Jzgknw, Colombia Colombia, 9 enero de (2019)

Duarte, L.C., F. Carvalheiro y otros cuatro autores, Comparison of Two Posthydrolysis Processes of Brewery's Spent Grain Autohydrolysis Liquor to Produce a Pentose-Containing Culture Medium, Applied Biochemistry and Biotechnology, 113$116,1041-58113(2004)$

El-Naggar, N.E.A., S. Deraz y A. Khalil, Bioethanol Production from Lignocellulosic Feedstocks based on Enzymatic Hydrolysis: Current Status and Recent Developments, doi: 10.3923/biotech.2014.1.21, Biotechnology, 13(1), 1-21, (2014)

Ghatak, H. R., Biorefineries from the Perspective of Sustainability: Feedstocks, Products, and Processes, Renewable and Sustainable Energy Reviews, 15(8), 4042-4052 (2011)

Jeoh, T., M. J. Cardona y otros tres autores, Mechanistic Kinetic Models of Enzymatic Cellulose Hydrolysis A review, doi:10.1002/bit.26277, Biotechnology and Bioengineering, 114, 1369-1385 (2017)

Liang, S. y C. Wan, Carboxylic Acid Production from Brewer's Spent Grain Via Mixed Culture Fermentation, Bioresource Technology, 182, 179-183 (2015)

Lynch, K. M., E. J. Steffen y E. K. Arendt, Brewers' Spent Grain: A Review with an Emphasis on Food and Health, doi: 10.1002/jib.363, Journal of the Institute of Brewing, 553-568 (2016)

Macheiner, D., B. F. Adamitsch, F. Karner y W. A. Hampel, Pretreatment and Hydrolysis of Brewer's Spent Grains, Engineering in Life Sciences, 3(10), 401-405 (2003)

McCarthy, A. L., Y. C. O'Callaghan y otros tres autores, Brewers' Spent Grain; Bioactivity of Phenolic Component, its role in Animal Nutrition and Potential for Incorporation in Functional Foods: a review, Proceedings of the Nutrition Society, 72(1), 117-125 (2013)

Miller, G.L., Use of Dinitrosalicylic Acid Reagent for Determination of Reducing Sugar, Analytical Chemistry, 31 (3), 426 428 (1959)

Mussatto, S. I., Brewer's Spent Grain: A Valuable Feedstock for Industrial Applications, doi: 10.1002/jsfa.6486, Journal of the Science of Food and Agriculture, 94(7), 1264-1275 (2014)

Mussatto, S. I., G. Dragone e I.C. Roberto, Brewers' Spent Grain: Generation, Characteristics and Potential Applications, doi: 10.1016/j.jcs.2005.06.001, Journal of Cereal Science, 43(1), 1-14 (2006)

Mussatto, S. I., J. Moncada, I. C. Roberto y C. A. Cardona, Techno-economic analysis for brewer's spent grains use on a biorefinery concept: The Brazilian case, doi.org/10.1016/j.biortech.2013.08.046, Bioresource Technology, 148, $302-310$ (2013)

Mussatto, S. I., M. Fernandes, A. M. F. Milagres e I. C. Roberto, Effect of hemicellulose and lignin on enzymatic hydrolysis of cellulose from brewer's spent grain, doi.org/10.1016/j.enzmictec.2007.11.006, Enzyme and Microbial Technology, 43(2), 124-129 (2008)

Niemi, P., C. Faulds y otros cuatro autores, Effect of a Milling Pre-treatment on the Enzymatic Hydrolysis of Carbohydrates in Brewer's Spent Grain, Bioresource Technology, 116, 155-160 (2012)

Pierre, G., F. Sannier y otros cinco autores, Evaluation of Thermomechanical Pretreatment for Enzymatic Hydrolysis of Pure Microcrystalline Cellulose and Cellulose from Brewers' Spent Grain, doi: 10.1016/j.jcs.2011.06.004, Journal of Cereal Science. Elsevier Ltd, 54(3), 305-310 (2011)

Plaza, P. E., L. J. Gallego-Morales y otros cuatro autores, Biobutanol production from brewer's spent grain hydrolysates by Clostridium beijerinckii, doi.org/10.1016/j.biortech.2017.07.139, Bioresource. Technology, 244, 166-174 (2017)

ProChile, (Ministerio de Relaciones Exteriores), Estudio de Mercado de Cerveza en Colombia, en línea, 9 enero 2019 , https://bit.ly/2xCPPcs, Chile (2011)

Raj, K. y C. Krishnan, Industrial Crops \& Products High Sugar Yields from Sugarcane (Saccharum officinarum) Bagasse using Low-Temperature Aqueous Ammonia Pretreatment and Laccase-Mediator assisted Enzymatic Hydrolysis, doi: 10.1016/j.indcrop.2017.11.047, Industrial Crops \& Products, Elsevier, 111, 673-683 (2018)

Ravindran, R. y A. Jaiswal, Microbial Enzyme Production Using Lignocellulosic Food Industry Wastes as Feedstock: A Review, doi: 10.3390/bioengineering3040030, Bioengineering, 3(4), 30 (2016)

Ravindran, R., G.A. Williams y A.K. Jaiswal, Bioresource Technology Reports Evaluation of brewer's spent grain hydrolysate as a substrate for production of thermostable $\alpha$-amylase by Bacillus stearothermophilus, Bioresource. Technology, 5, (January), 141-149 (2019)

Ravindran, R., S. Jaiswal, N. Abu-Ghannam y A. K. Jaiswal., A Comparative Analysis of Pretreatment Strategies on the Properties and Hydrolysis of Brewers' Spent Grain, doi: 10.1016/j.biortech.2017.06.039, Bioresource Technology, Elsevier Ltd, 248, 272-279 (2018)

TAPPI. Test Methods T203 cm-99, Technical Association of the Pulp and Paper Industry. Atlanta, USA (1999)

TAPPI. Test Methods T222 cm-02T, Technical Association of the Pulp and Paper Industry. Atlanta, USA (2009) 
Van Dyk, J.S. y B.I. Pletschke, A Review of Lignocellulose Bioconversion Using Enzymatic Hydrolysis and Synergistic Cooperation between Enzymes - Factors Affecting Enzymes, Conversion and Synergy, doi: 10.1016/j.biotechadv.2012.03.002, Biotechnology Advances. Elsevier Inc., 30(6), 1458-1480 (2012)

Venkata Mohan, S., G. N. Nikhil y otros cinco autores, Waste biorefinery models towards sustainable circular bioeconomy: Critical review and future perspectives, Bioresource Technology, 215, 2-12 (2016)

Wilkinson, S., K. A. Smart y D. J. Cook, Optimising the (Microwave) Hydrothermal Pretreatment of Brewers Spent Grains for Bioethanol Production, doi: 10.1155/2015/369283, Journal of Fuels, 2015, 369283 (2015)

Wilkinson, S., K. A. Smart, S. James y D. J. Cook, Bioethanol Production from Brewers Spent Grains Using a Fungal Consolidated Bioprocessing (CBP) Approach, doi: 10.1007/s12155-016-9782-7, Bioenergy Research, 10(1) (2017)

Xiros, C. y P. Christakopoulos, Biotechnological Potential of Brewers Spent Grain And its Recent Applications, doi: 10.1007/s12649-012-9108-8, Waste and Biomass Valorization, 3(2), 213-232 (2012)

Xiros, C., E. Topakas, P. Katapodis y P. Christakopoulos, Evaluation of Fusarium oxysporum as an enzyme factory for the hydrolysis of brewer's spent grain with improved biodegradability for ethanol production, Industrial Crops and Products, 28(2), 213-224 (2008)

Zabed, H., J. Sahu, A. Boyce y G. Faruq, Fuel ethanol production from lignocellulosic biomass: An overview on feedstocks and technological approaches, doi: 10.1016/j.rser.2016.08.038, Renewable and Sustainable Energy Reviews, Elsevier, 66, 751-774 (2016)

Zhang, J. y L. Zang, Enhancement of biohydrogen production from brewers' spent grain by calcined-red mud pretreatment, Bioresource Technology 209, 73-79 (2016)

Zhao, H., J.H. Kwak y otros cuatro autores, Effects of crystallinity on dilute acid hydrolysis of cellulose by cellulose ballmilling study. Energy Fuels 20, 807-811 (2006) 
